\title{
COVID-19 and cardiovascular disease
}

\author{
Indrakumar $\mathbf{J}^{1}$, Wijesinghe $\mathbf{R}$ A $\mathbf{N} \mathbf{K}^{2}$ \\ Journal of the Ceylon College of Physicians, 2020, 51, 26-30
}

Key words: COVID-19, cardiac, pulmonary embolism, arterial thrombosis

\section{Introduction}

Currently, the world is experiencing a pandemic of COVID-19 corona virus infection, which is still evolving and the number of cases continue to rise at an exponential rate. It is difficult to predict the future course of this disease at this point in time.

Initial reports show that significant majority of the patients (81\%) with COVID-19 infection experience mild symptoms like dry cough or a sore throat. About $14 \%$ have severe symptoms with dyspnoea, desaturation, tachypnoea, with significant (>50\%) lung infiltrates and only $5 \%$ have critical infection, which is defined as having respiratory failure, septic shock and multiorgan failure $^{1}$. We are aware from the previous coronavirus and influenza epidemics that apart from their predominant respiratory involvement, there can be cardiac involvement leading to cardiac complications such as acute coronary syndromes ${ }^{2}$, arrhythmias ${ }^{3}$, and exacerbation of heart failure ${ }^{4}$. Although many cardiovascular effects of COVID-19 are unfolding, their severity, extent, short- and long-term effects are still unknown.

\section{Comorbidities in patients with COVID-19}

Cardiovascular diseases (CVD) were a common comorbidity in previous corona virus infections namely, severe acute respiratory syndrome (SARS) and middle east respiratory syndrome (MERS) epidemics. In SARS, diabetes and cardiovascular diseases were seen in $11 \%$ and $8 \%$ of the patients respectively and they had increased risk of death by twofold ${ }^{5}$. Whereas in MERS diabetes and hypertension were seen in about $50 \%$ and cardiovascular diseases in $30 \%$ of patients ${ }^{6}$.
The mechanisms of cardiac injury are likely to be caused by increased stress on the heart by respiratory failure and hypoxaemia, direct myocardial injury by the virus and indirect injury due to systemic hyperinflammatory response (cytokine storm) (Figure 1). Chronic cardiac diseases may become unstable due to increased metabolic demand due to the viral infection and the systemic inflammation in the setting of a compromised cardiac reserve. Systemic inflammation also produces a procoagulant state ${ }^{7}$ increasing the risk of myocardial infarction and stent thrombosis in patients who had recent coronary interventions.

Most data come from China, the birth place of COVID-19 where in a cohort of 191 COVID-19 patients almost half (48\%) had comorbidities, which was significantly more in non-survivors (67\%). Hypertension was seen in $30 \%$ ( $48 \%$ of non survivors), diabetes in $19 \%$ (31\% of non survivors) and CVD 8\% (31\% of non survivors) ${ }^{8}$ suggesting that these conditions are associated with worse prognosis. In non-survivors the high-sensitivity troponins (hs-CTI) and inflammatory biomarkers (ferritin and Interleukin-6) were significantly elevated and had higher incidence of heart failure $(52 \%$ vs $12 \%$ in survivors) and acute cardiac injury (59\% vs $1 \%$ in survivors $)^{8}$. These findings suggest high inflammatory burden and possibly, myocarditis contribute to the poor prognosis. In another study, which analyzed 138 hospitalized COVID-19 patients also showed similar results with hypertension in $31 \%$ (58\% of those in ICU care), diabetes in $10 \%$ (22\% of those in ICU care), and CVD in $15 \%$ (25\% of those in ICU care) ${ }^{9}$. A meta-analysis of about 46,000 infected patients shows that hypertension was the most prevalent comorbidity $(17 \pm 7 \%)$, followed by diabetes ( $8 \pm 6 \%)$, cardiovascular

\footnotetext{
${ }^{1}$ Faculty of Medical Sciences, University of Sri Jayewardenepura, Sri Lanka, ${ }^{2}$ General Sir John Kotelawala Defence University, Sri Lanka.
}

Correspondence: JI, e-mail: indrak2004@gmail.com

http://orcid.org/0000-0002-1916-5409

Received 02 May 2020, accepted 20 May 2020.

This is an open-access article distributed under the terms of the Creative Commons Attribution License, which permits unrestricted use, distribution, and reproduction in any medium, provided the original author and source are credited. 
diseases $(5 \pm 4 \%)$ compared to a remarkably low underline respiratory diseases $(2 \pm 0 \%)^{10}$. Weather the observed high prevalence of hypertension is due to a reaction to the illness or an underline co-morbid condition is not clear.

\section{Myocardial injury in COVID-19}

Myocardial infiltration by mononuclear inflammatory cells in COVID-19 suggests that this virus affects myocardium and cause myocarditis and systolic dysfunction ${ }^{11}$. Myocardial injury (elevated troponins) is highly prevalent (20\%) in hospitalized patients with COVID-19 infection who are more commonly men, older age, have comorbidities (hypertension, coronary heart disease, cardiomyopathy and chronic kidney disease) and among patients who are more likely to develop complications such as acute kidney injury and ARDS ${ }^{12,13}$. Furthermore, patients with myocarditis are more likely to require both noninvasive (46\%) and invasive mechanical (22\%) ventilation and have higher risk of in-hospital mortality (51\%). Another study from China reported that $12 \%$ of patients without any cardiovascular disease had evidence of myocarditis suggested by elevated troponins or cardiac arrest during hospital stay ${ }^{14}$. About half the non survivors (46\%) had raised high sensitivity troponin T as opposed to $1 \%$ of survivors ${ }^{8}$. In another study regarding factors associated with outcomes in hospitalized patients,
35\% had CVD (hypertension coronary artery disease or cardiomyopathy) and $28 \%$ had features of myocardial injury (elevated troponin) ${ }^{13}$. The mortality was very high $(60 \%)$ in those with raised troponins when compared to those with normal troponins $(9 \%)^{13}$. Patients with elevated troponins also showed higher risks of ARDS, malignant arrhythmias, acute renal injury and coagulopathy ${ }^{13}$.

There appears to be 2 patterns of myocardial injury due to COVID-19. In those patients presenting with typical symptoms, the hs-cTI were elevated ( $8.8 \mathrm{pg} / \mathrm{L})$ in non survivors on day 4 when compared to those of survivors $(2.5 \mathrm{pg} / \mathrm{L})^{8}$. Thereafter, the troponins remained more or less same (2.5-4.4 pg/L) in survivors but continued to rise steadily to $290 \mathrm{pg} / \mathrm{L}$ on day 22 in non-survivors ${ }^{8}$. There has been an associated rise of inflammatory markers (e.g. D-dimer, ferritin, interleukin-6, lactase dehydrogenase) suggesting the probability of development of cytokine storm or a secondary haemophagocytic lympohistiocytosis rather than a simple myocarditis. The cytokine storm, or systemic hyperinflammation, is a feature of advanced stage (Figure 2) characterized by multiorgan dysfunction and elevation of typical inflammatory markers. This can result in fulminant myocarditis and acute respiratory distress syndrome causing high mortality. Also, profound hypoxia may induce excessive intracellular

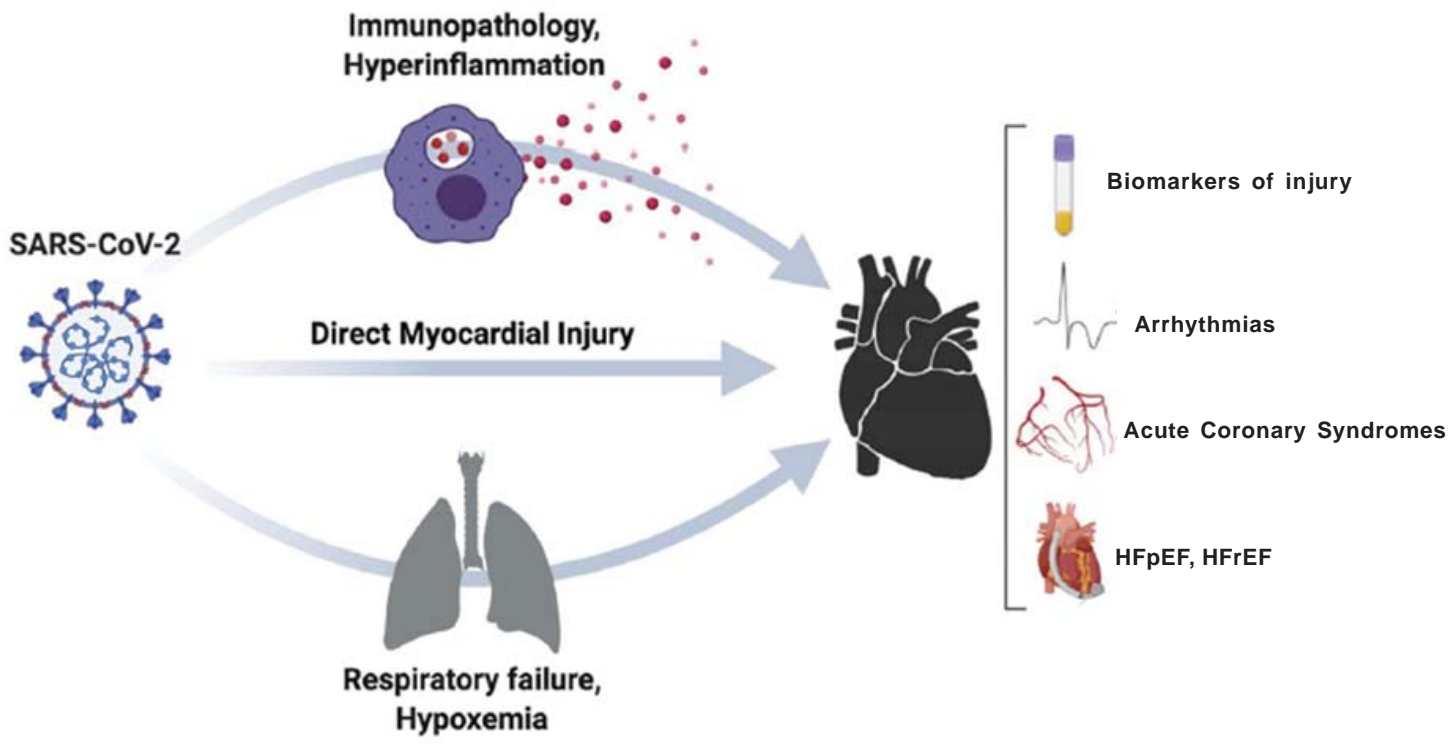

Figure 1. Mechanisms of cardiac injury and its consequences.

COVID-19 and the Heart. Akbarshakh Akhmerov and Eduardo Marban. Originally published 7 Apr 2020 https://doi.org/ 10.1161/CIRCRESAHA.120.317055 Circulation Research.

(HFpEF - heart failure with preserved ejection fraction, HFrEF - heart failure with reduced ejection fraction) 


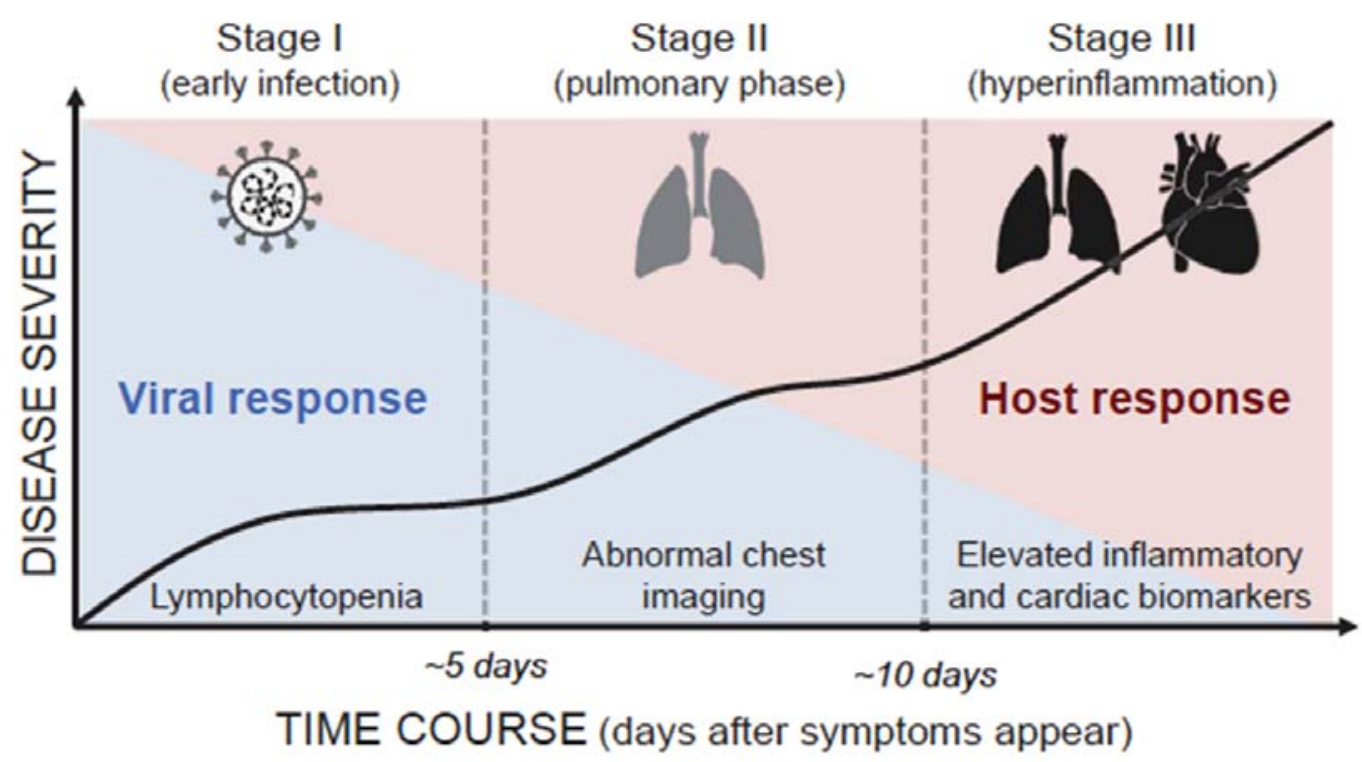

Figure 2. Progression of the disease in acute COVID-19.

COVID-19 and the Heart. Akbarshakh Akhmerov and Eduardo Marban. Originally published 7 Apr 2020 https://doi.org/ 10.1161/CIRCRESAHA.120.317055 Circulation Research.

calcium resulting in apoptosis of cardiac myocytes ${ }^{14}$. The other pattern is suggested in patients presenting with predominantly cardiac symptoms who develop an isolated stress cardiomyopathy or viral myocarditis ${ }^{15}$.

\section{NT-proBNP and COVID-19}

It has been known that an elevated NT-proBNP is a strong and independent predictor of mortality in community-acquired pneumonia ${ }^{16}$. Furthermore, a recent study has been shown that high plasma NTproBNP levels (> $88.64 \mathrm{pg} / \mathrm{mL}$ ) is associated with increased risk of in-hospital death in severe COVID19 patients $^{17}$. They were mostly older patients with increased levels of markers of cardiac injury and systematic inflammation.

\section{Arrythmias}

COVID-19 can also cause tachyarrhythmias, bradyarrhythmias and asystole. Palpitations occur in about $7 \%$ and much higher percentage (17\%) of critically ill patients develop arrhythmia the character of which is not yet described ${ }^{9,18}$.

There is a strong association between viral respiratory infections and acute myocardial infarction in influenza epidemics ${ }^{19}$ and SARS epidemics ${ }^{20}$. Acute coronary syndromes and acute myocardial infarctions can occur in COVID-19 patients although the incidence of such complications are not yet known. However, it is important know that the high incidence of acute cardiac injury can be mistakenly diagnosed as ACS due to the presence of the ECG abnormalities, troponin elevations and chest pain in these patients.

\section{ACEI and ARB in COVID-19}

Angiotensin-converting enzyme 2 (ACE2) is seen in lung alveolar cells, heart, kidney and gut. It converts angiotensin II into angiotensin-(1-7), I, which is an inactive form that diminishes vasoconstriction mediated by the renin-angiotensin system. SARS-CoV spike protein binds to this enzyme receptor ACE2 to enter the alveolar cells and once enters it down regulates the receptors resulting in increased levels of angiotensin II, which causes myocardial dysfunction ${ }^{21}$. This can be another mechanism of cardiac dysfunction in COVID-19 infection. Also, as ACE2 receptors are highly expressed in the heart, the virus can enter into myocardium to cause myocardial infection in the same manner. This phenomenon of viral entry to alveoli and heart being facilitated by ACE2 has led to the controversy regarding the use of angiotensin converting enzyme inhibitors or angiotensin receptor blockers in COVID-19 infected patients. Based on the theoretical explanation it has been suggested that these drugs could increase both the risk and the severity of infection. 
However, this assumption about the safety of these drugs has not been based on any scientific evidence to support its harmful effects. As such the Council on hypertension of the European Society of Cardiology in its latest position statement strongly recommend that these drugs should be continued if the patient is already taking and should not be discontinued because of the COVID-19 infection ${ }^{22}$.

\section{Thromboembolic complications}

COVID-19 infection has a high risk of both venous and arterial thromboembolism most likely due to excessive inflammation, hypoxia, immobilization and diffuse intravascular coagulation. Despite being on standard thromboprophylaxis about $31 \%$ of patients, in the intensive care units, developed thrombotic complications $(27 \%$ venous thromboembolism and $3.7 \%$ arterial thrombosis $)^{23}$.

\section{Long term effects of COVID-19}

Cardiovascular complications are known to occur during short term and long term (10 years) following resolution of pneumonia due to persistence of systemic inflammatory and procoagulant activity even after the resolution of initial illness ${ }^{24,25}$. This can also be applicable to patients who have recovered from COVID-19 infections as cardiovascular complications can still occur during convalescence or in the long term. A report from Italy describes a case of fulminant myocarditis one week after recovery of respiratory symptoms suggesting the persistence of inflammation after recovery ${ }^{26}$. Follow up studies in those who have recovered are therefore important.

\section{Conclusions}

Cardiovascular complications of COVID-19 are common and have high risk of morbidity and mortality. Preexisting cardiovascular disease and advanced age contribute to adverse outcomes. Myocardial injury can present as acutely, associated with cardiac dysfunction at presentation, or can develop as the disease progress to a stage, where the hyperinflammation or cytokine storm develop, causing increased mortality. As the data on COVID-19 is still evolving, some guidance can be drawn on current knowledge of cardiovascular complications of other similar viruses like influenza, SARS and MERS. Follow up studies on survivors are necessary to assess the long-term impact of this virus on cardiovascular system.

\section{References}

1. Wu Z, McGoogan JM. Characteristics of and Important Lessons from the Coronavirus Disease 2019 (COVID-19) Outbreak in China: Summary of a Report of 72-314 Cases from the Chinese Center for Disease Control and Prevention. Journal of the American Medical Association 2020 Feb 24. doi: 10.1001/jama.2020.2648. [Epub ahead of print]

2. Kwong JC, Schwartz KL, Campitelli MA. Acute myocardial infarction after laboratory-confirmed influenza infection. New England Journal of Medicine 2018; 378(4): 345-53.

3. Madjid M, Connolly AT, Nabutovsky Y, Safavi-Naeini P, Razavi $\mathrm{M}$, Miller CC. Effect of high influenza activity on risk of ventricular arrhythmias requiring therapy in patients with implantable cardiac defibrillators and cardiac resynchronization therapy defibrillators. American Journal of Cardiology 2019; 124(1): 44-50.

4. Kytömaa S, Hegde S, Claggett B, et al. Association of Influenza-like Illness Activity With Hospitalizations for Heart Failure: The Atherosclerosis Risk in Communities Study. JAMA Cardiology 2019 Apr 1; 4(4): 363-9.

5. Chan JWM, Ng CK, Chan $\mathrm{YH}$, et al. Short term outcome and risk factors for adverse clinical outcomes in adults with severe acute respiratory syndrome (SARS). Thorax 2003; 58: 686-9.

6. Badawi A and Ryoo SG. Prevalence of comorbidities in the Middle East respiratory syndrome coronavirus (MERS-CoV): a systematic review and meta-analysis. International Journal of Infectious Diseases 2016; 49: 129-133.

7. Libby P, Simon DI. Inflammation and thrombosis: the clot thickens. Circulation 2001; 103: 1718-20.

8. Zhou F, Yu T, Du R, et al. Clinical course and risk factors for mortality of adult inpatients with COVID-19 in Wuhan, China: a retrospective cohort study. Lancet 2020; 395(10229): 1054-62.

9. Wang D, Hu B, Hu C, et al. Clinical Characteristics of 138 Hospitalized Patients With 2019 Novel Coronavirus-Infected Pneumonia in Wuhan, China. The Journal of the American Medical Association 2020; 323: 1061-9.

10. Yang J, Zheng Y, Gou X, et al. Prevalence of comorbidities in the novel Wuhan coronavirus (COVID-19) infection: a systematic review and meta-analysis. International Journal of Infectious Diseases 2020 Mar 12. pii: S12019712(20)30136-3. doi: 10.1016/j.ijid.2020.03.017. [Epub ahead of print]

11. Xu Z, Shi L, Wang Y, et al. Pathological findings of COVID-19 associated with acute respiratory distress syndrome. Lancet Respiratory Medicine 2020 Apr; 8(4): 420-22.

12. Shi S, Qin M, Shen B, et al. Cardiac Injury With Mortality in Hospitalized Patients With COVID-19 in Wuhan, China. JAMA Cardiology 2020 Mar 25. doi: 10.1001/jamacardio.2020.0950. [Epub ahead of print] 
13. Guo T, Fan Y, Chen M, et al. Cardiovascular Implications of Fatal Outcomes of Patients With Coronavirus Disease 2019 (COVID-19). JAMA Cardiology 2020 Mar 27. doi: 10.1001/ jamacardio.2020.1017. [Epub ahead of print]

14. Zheng Y-Y, Ma Y-T, Zhang J-Y and Xie X. COVID-19 and the cardiovascular system. Nature Reviews Cardiology. March 5, 2020. doi: 10.1038/s41569-020-0360-5. [epub ahead of print].

15. Hu H, Ma F, Wei X, Fang Y. Coronavirus fulminant myocarditis saved with glucocorticoid and human immunoglobulin. European Heart Journal. March 16, 2020. doi: 10.1093/ eurheartj/ehaa190. [epub ahead of print]

16. Jeong KY, Kim K, Kim TY, Lee CC, Jo SO, Rhee JE, Jo YH, Suh GJ, Singer AJ. Prognostic value of $\mathrm{N}$-terminal pro-brain natriuretic peptide in hospitalised patients with communityacquired pneumonia. Emergency Medicine Journal 2011 Feb; 28(2): 122-7.

17. Gao L, Jiang D, Wen, X, et al. Prognostic value of NTproBNP in patients with severe COVID-19. Respiratory Research 2020; 21, 83. https://doi.org/10.1186/s12931-02001352

18. Liu K, Fang YY, Deng Y, et al. Clinical characteristics of novel coronavirus cases in tertiary hospitals in Hubei Province. Chinese Medical Journal 2020 Feb 7. doi: $10.1097 /$ CM9.0000000000000744. [Epub ahead of print]

19. Kwong JC, Schwartz KL, Campitelli MA. Acute myocardial infarction after laboratory-confirmed influenza infection. New England Journal of Medicine 2018; 378: 345-53.

20. Peiris JS, Chu CM, Cheng VC, et al. HKU/UCH SARS Study
Group. Clinical progression and viral load in a community outbreak of coronavirus-associated SARS pneumonia: a prospective study. Lancet 2003; 24: 361(9371): 1767-72.

21. Oudit GY, Kassiri Z, Jiang C, et al. SARS - coronavirus modulation of myocardial ACE2 expression and inflammation in patients with SARS. European Journal of Clinical Investigation 2009; 39: 618-25.

22. 19.1. Position Statement of the ESC council on Hypertension on ACE-inhibitors and Angiotensin Receptor Blockers. 13 March 2020. www.escardio.org/Councils/CouncilonHypertension-(CHT)/News/position-statement-of-theesccouncil-on-hypertension-on-ace-inhibitors-and-ang.

23. Klok FA, Kruip MJHA, van der Meer NJM, et al. Incidence of thrombotic complications in critically ill ICU patients with COVID-19. Thrombosis Research 2020 Apr 10. pii: S00493848(20)30120-1. doi: 10.1016/j.thromres.2020.04.013. [Epub ahead of print]

24. Corrales-Medina VF, Alvarez KN, Weissfeld LA, et al. Association between hospitalization for pneumonia and subsequent risk of cardiovascular disease. Journal of the American Medical Association 2015; 313: 264-74.

25. Cangemi R, Calvieri C, Falcone M, et al. SIXTUS Study Group. Relation of Cardiac Complications in the Early Phase of Community-Acquired Pneumonia to Long-Term Mortality and Cardiovascular Events. American Journal of Cardiology 2015; 15: 116(4): 647-51.

26. Inciardi RM, Lupi L, Zaccone G, et al. Cardiac Involvement in a Patient with Coronavirus Disease 2019 (COVID-19). JAMA Cardiology 2020 Mar 27. doi: 10.1001/jamacardio.2020.1096. [Epub ahead of print] 